\title{
On a Power Transformation of Half-Logistic Distribution
}

\author{
S. D. Krishnarani \\ Department of Statistics, Farook College, Kozhikode, Kerala 673632, India \\ Correspondence should be addressed to S. D. Krishnarani; krishnaranisd@gmail.com
}

Received 7 September 2015; Revised 22 December 2015; Accepted 24 December 2015

Academic Editor: Aera Thavaneswaran

Copyright (C) 2016 S. D. Krishnarani. This is an open access article distributed under the Creative Commons Attribution License, which permits unrestricted use, distribution, and reproduction in any medium, provided the original work is properly cited.

\begin{abstract}
A new continuous distribution on the positive real line is constructed from half-logistic distribution, using a transformation and its analytical characteristics are studied. Some characterization results are derived. Classical procedures for the estimation of parameters of the new distribution are discussed and a comparative study is done through numerical examples. Further, different families of continuous distributions on the positive real line are generated using this distribution. Application is discussed with the help of real-life data sets.
\end{abstract}

\section{Introduction}

Distributions defined on the positive real line are widely used in modeling of survival data. The Weibull, Pareto, and Exponential distributions have major roles in fitting data sets in the fields of computer science, engineering, biology, and so forth. Half-logistic distribution (HLD) is another life distribution used in reliability analysis by many researchers. The half-logistic random variable studied by Balakrishnan $[1,2]$ has survival function

$$
\bar{F}(x)=\frac{2}{1+e^{x}}, \quad x>0 .
$$

By imparting the location and scale parameters, its probability density function (pdf) is

$$
f(x ; \mu, \sigma)=\frac{2 e^{((x-\mu) / \sigma)}}{\sigma \cdot\left(1+e^{((x-\mu) / \sigma)}\right)^{2}}, \quad x \geq \mu, \sigma>0 .
$$

In the past few years many researchers have paid much attention to this distribution and several generalizations have been introduced. Srinivasa Rao et al. [3], Olapade [4-6], Cordeiro et al. [7], Kantam et al. [8], and so forth are some of the recent works in this area.

It is well known that through power transformation, the Weibull is an extension of exponential while the power function distribution is that of uniform. Hence, it is of interest to know what would be the distribution of similar power transformation of half-logistic distributions. Motivated by this, in the present paper, we introduce a new continuous distribution on the positive real line using a transformation of half-logistic random variable. We study the properties and applications of this so-called generalization of half-logistic distribution.

The remaining part of the paper is organized as follows. In Section 2, Power Half-Logistic Distribution is introduced and its properties are studied. In Section 3, some characterization results are derived. Estimation of the parameters is done in Section 4 and numerical illustrations are given therein. Section 5 deals with extensions of this new transformed distribution. Application to real data sets is considered in Section 6 followed by a concluding section at the end.

\section{Power Half-Logistic Distribution}

Let $Y$ be a random variable following half-logistic distribution with survival function

$$
\bar{F}_{Y}(y)=\frac{2}{1+e^{\beta y}}, \quad y>0, \beta>0 .
$$

Consider the transformation, $X=Y^{1 / \alpha}$. Then the survival function of $X$ is

$$
\bar{F}_{X}(x)=\frac{2}{1+e^{\beta x^{\alpha}}}, \quad x>0, \alpha, \beta>0
$$




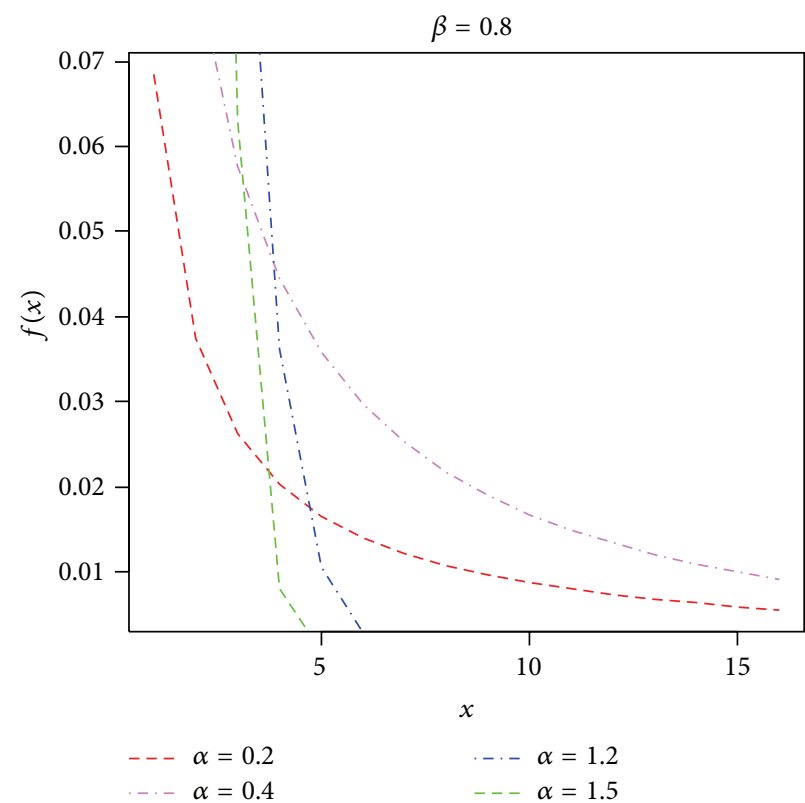

(a)

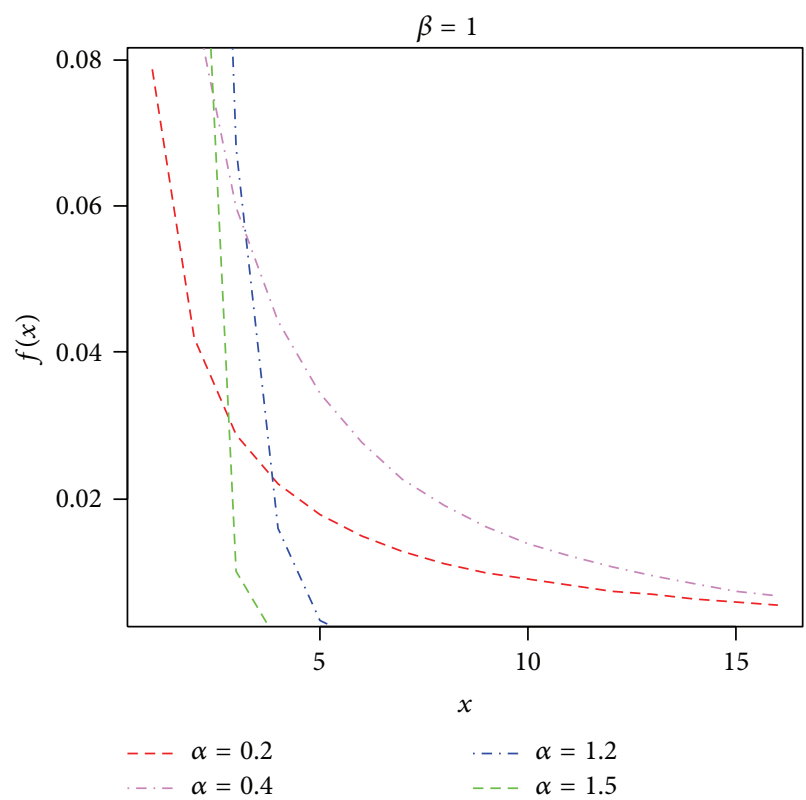

(b)

FIGURE 1: pdfs of PHLD for various values of the parameters.

and its pdf is

$$
f(x)=\frac{2 \alpha \beta e^{\beta x^{\alpha}} x^{\alpha-1}}{\left(1+e^{\beta x^{\alpha}}\right)^{2}}, \quad x>0, \alpha>0, \beta>0 .
$$

Hereinafter we call the random variable $X$ with pdf (5) as the Power Half-Logistic Distribution (PHLD).

The graphical form of the pdf for various values of $\alpha$ and $\beta$ is given in Figure $1(\beta=0.8$ and $\beta=1)$. When $\beta$ is fixed and $\alpha$ is increasing in $(0,1)$, the pdf becomes more convex. But when $\alpha$ is $>1$ it becomes concave. Another characteristic is that for $0<\alpha<1$, the distribution is heavy tailed but for $\alpha>1$, the distribution is light tailed as compared to HLD (see Figure 2). So it can be used to model data sets having tail probability less or greater than HLD. The plots of survival function and hazard rates for different values of $\alpha$ when $\beta=$ 0.5 and 1.5 are shown in Figures 3 and 4, respectively, for an alternate view of the behavior of the distribution.

Next we explore the analytical properties of the PHLD, deriving its moments, median, quantiles, hazard function, and $\log$ odds function, and summarize them below.

Properties. (1) The sth moment $E\left(X^{s}\right)=\left(2 s /\left(\beta^{s} \cdot \alpha\right)\right) \Gamma(s /$

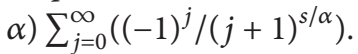

(2) Median $=((\log 3) / \beta)^{1 / \alpha}$.

(3) The $p$ th quantile is $((1 / \beta) \log ((1+p) /(1-p)))^{1 / \alpha}$.

(4) Hazard rate $r(x)=\alpha \beta e^{\beta x^{\alpha}} x^{\alpha-1} /\left(1+e^{\beta x^{\alpha}}\right)$. It can be seen in Figure 4 that for $\alpha>1$, the distribution has increasing failure rate (IFR), but for $0<\alpha<1$ the distribution has decreasing failure rate (DFR).

(5) The log odds function is $\log \left[\left(e^{\beta x^{\alpha}}-1\right) / 2\right]$.

It may be noted that in a recent study on the exponentiated half-logistic family by Cordeiro et al. [7] a special case of it called half-logistic Weibull distribution has been just mentioned without any elaborate study. They proposed a new exponentiated half-logistic (EHL) family as a competitive alternative for lifetime data analysis. For any parent continuous distribution $G$ they defined the corresponding EHL$G$ distribution with distribution function $\left[\left(1-\bar{G}(x)^{\lambda}\right) /(1+\right.$ $\left.\left.\bar{G}(x)^{\lambda}\right)\right]^{\alpha}$. This new family extends several common distributions such as Frechet, normal, log-normal, Gumbel, and loglogistic distributions. It is interesting to observe that PHLD is the same as the distribution pointed out there when $G(x)=$ $1-\exp \left(-x^{\beta}\right)$.

Next we derive some characterization results of PHLD.

\section{Characterizations}

In the first characterization we establish a relationship between the PHLD and Weibull distribution.

Result 1. Suppose $\bar{F}(x)$ and $\bar{G}(x)$ are survival functions with respective pdfs $f$ and $g$. Then in the equation

$$
\frac{d}{d x}\left(\frac{1}{\bar{G}(x)}\right)=\frac{1}{2} \frac{d}{d x}\left(\frac{1}{\bar{F}(x)}\right)
$$

or

$$
\frac{g(x)}{(\bar{G}(x))^{2}}=\frac{1}{2} \frac{f(x)}{(\bar{F}(x))^{2}},
$$

$g(x)$ has PHLD if, and only if, $f(x)$ is Weibull.

Proof. Suppose the pdf $g(x)$ is PHLD with the form as in (5) and then substituting in (7) and further on integrating, we get

$$
\bar{F}(x)=e^{-\beta x^{\alpha}},
$$

which is the survival function of Weibull random variable. 


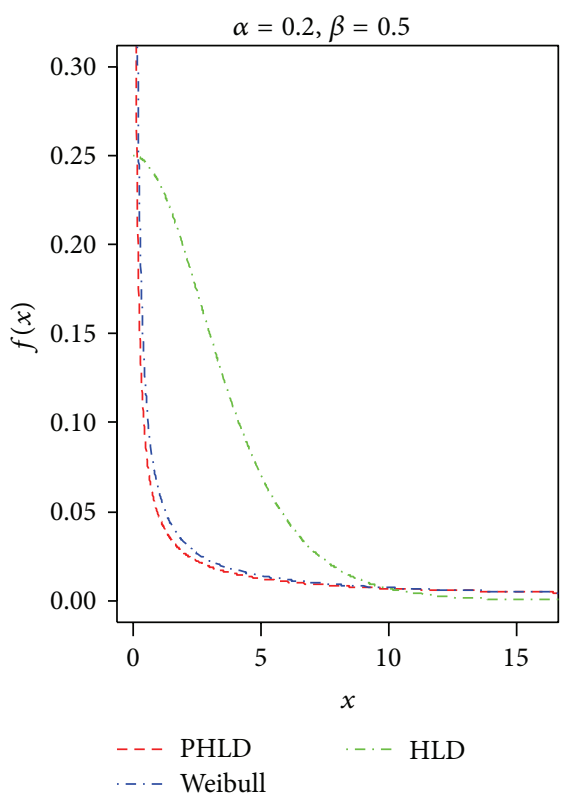

(a)

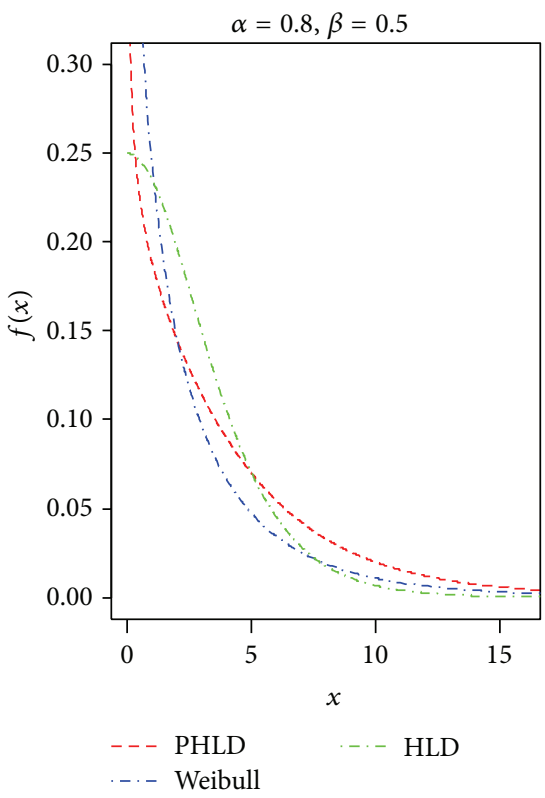

(b)

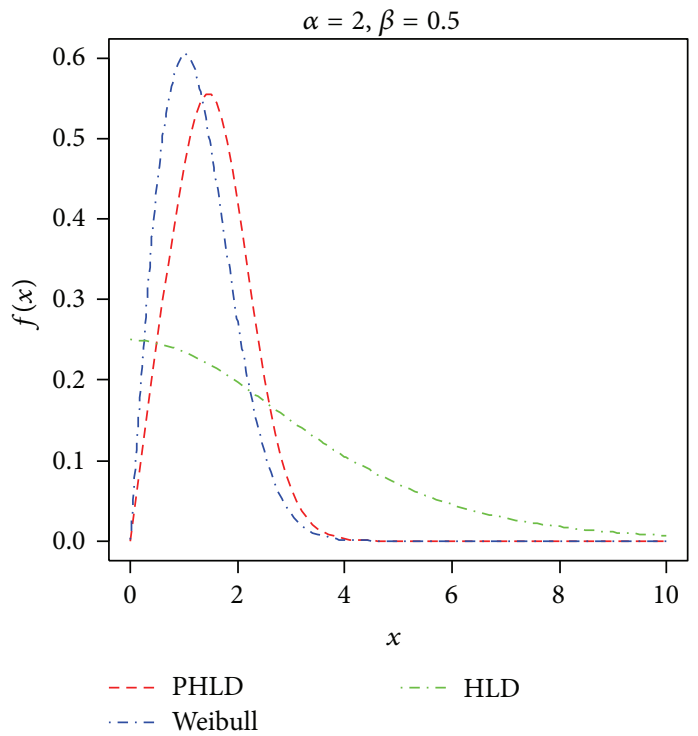

(c)

Figure 2: Comparison of PHLD, HLD, and Weibull densities.

Conversely assuming $f(x)$ as Weibull with pdf

$$
f(x)=e^{-\beta x^{\alpha}} \alpha \beta x^{\alpha-1}
$$

and substituting in (7), we get

$$
\bar{G}(x)=\frac{2}{1+e^{\beta x^{\alpha}}},
$$

which is the survival function of PHLD.

Result 2. The function $g(x)$ in

$$
x^{1 / \alpha} \frac{g\left(x^{1 / \alpha}\right)}{x}=\alpha f(x)
$$

is the pdf of PHLD if, and only if, $f(x)$ is the pdf of HLD.
Proof. The proof easily follows.

Result 3. For a survival function $\bar{G}(x)$, the functional equation

$$
f\left(\left(x^{\alpha}+y^{\alpha}\right)^{1 / \alpha}\right)=f(x) f(y)
$$

(a variant of Cauchy's equation) is satisfied by $f(x)=2 /$ $\bar{G}(x)-1$ if, and only if, $\bar{G}(x)=2 /\left(1+e^{c x^{\alpha}}\right)$.

Proof. Suppose $f(x)=2 / \bar{G}(x)-1$ satisfies the given functional equation.

Then there exists a constant $c$ such that $f(x)=e^{c x^{\alpha}}$ (see [9]). Hence, $\bar{G}(x)=2 /\left(1+e^{c x^{\alpha}}\right)$. 


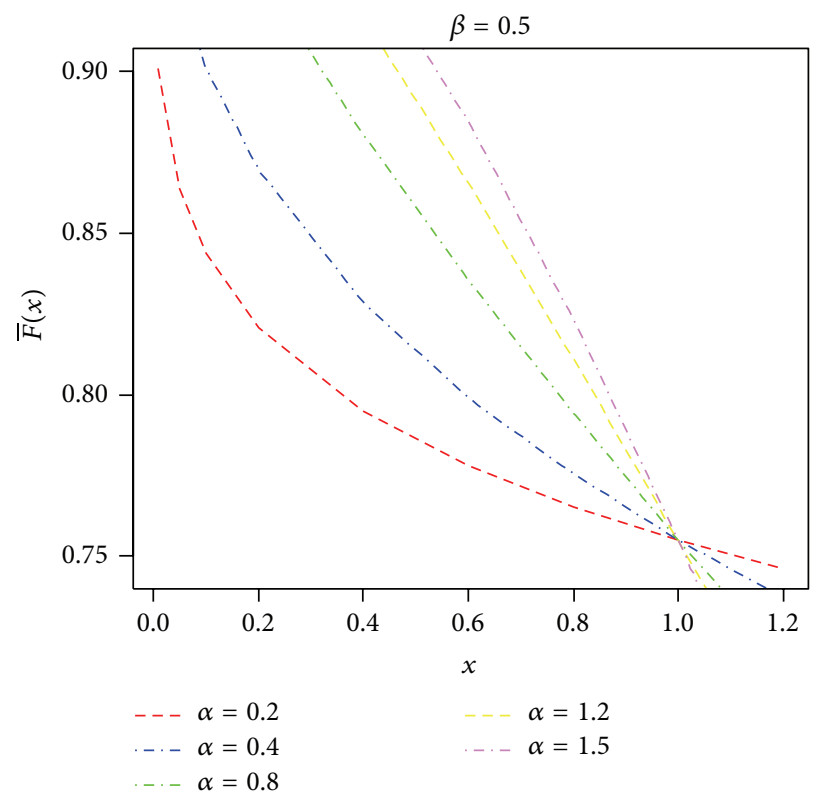

(a)

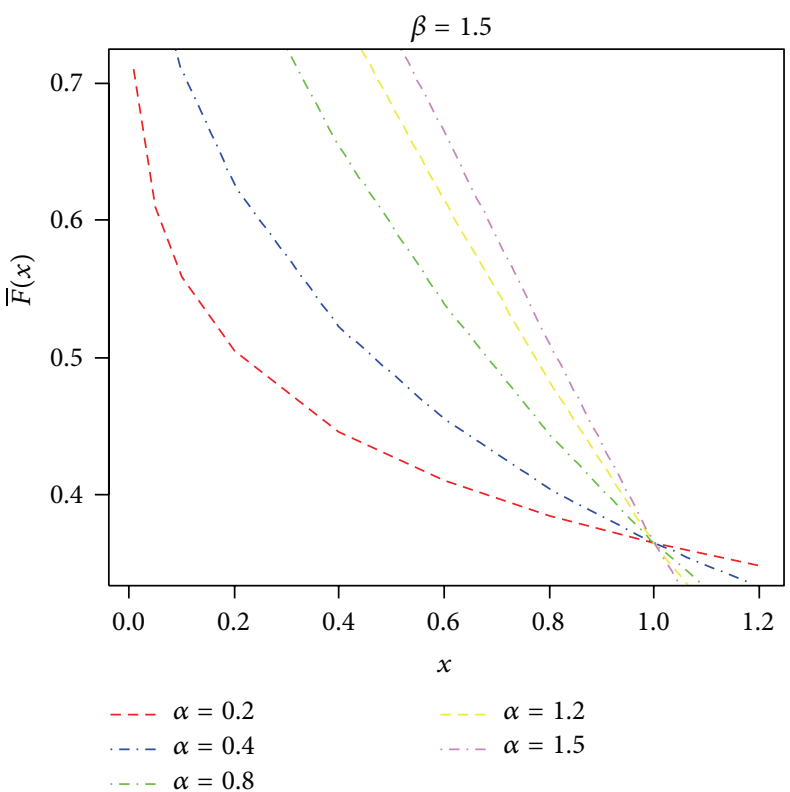

(b)

FIGURE 3: Survival function of PHLD ( $\beta=0.5$ and 1.5).

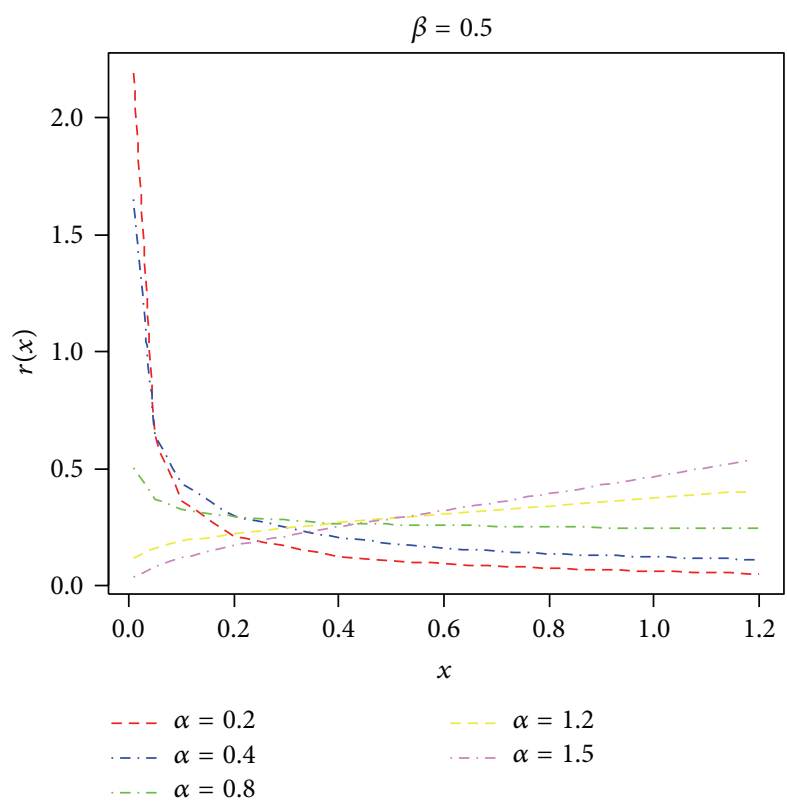

(a)

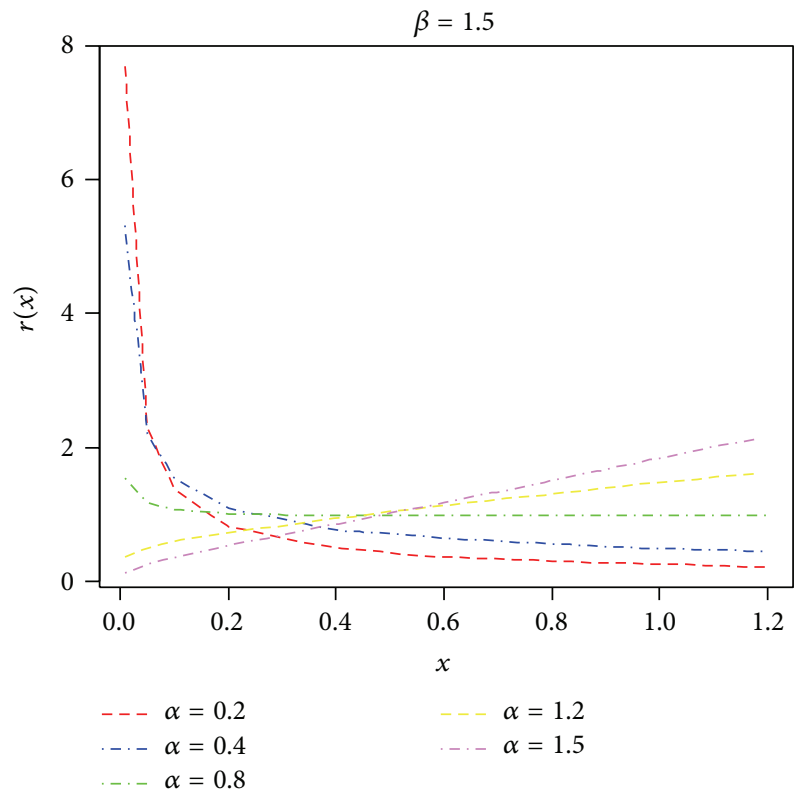

(b)

FIgURE 4: Hazard rate of PHLD ( $\beta=0.5$ and 1.5). $\left.e^{c x^{\alpha}}\right)$

Converse part easily follows by assuming $\bar{G}(x)=2 /(1+$

One may derive further characterizations of PHLD by taking the $(1 / \alpha)$ th power of half-logistic variables in the results of Olapade [4], which described some characterizations of half-logistic distribution.

\section{Estimation of the Parameters}

We use the following three common methods for estimation purpose. Numerical illustrations are also done subsequently.

4.1. Maximum Likelihood Estimation. Suppose a sample of size $n$ is taken from PHLD with density function (5). By taking logarithms and finding the derivative with respect to $\alpha$ 
and $\beta$ we have two nonlinear equations which can be solved simultaneously and numerically.

We have

$$
\begin{aligned}
\frac{\partial \log L}{\partial \alpha}= & \frac{n}{\alpha}+\sum_{i=1}^{n} \beta x_{i}^{\alpha} \log x_{i}+\sum_{i=1}^{n} \log x_{i} \\
& -2 \sum_{i=1}^{n} \frac{1}{1+e^{\beta x_{i}^{\alpha}}} \log x_{i} x_{i}^{\alpha} \beta e^{\beta x_{i}^{\alpha}}, \\
\frac{\partial \log L}{\partial \beta}= & \frac{n}{\beta}+\sum_{i=1}^{n} x_{i}^{\alpha}-\frac{2}{1+e^{\beta x_{i}^{\alpha}} x_{i}^{\alpha}} .
\end{aligned}
$$

4.2. Method of Moments. Method of moment estimation is another common method used for estimation of parameters. Equating the first and second raw moments to corresponding central moments, the following are the equations obtained:

$$
\begin{aligned}
& \frac{\sum_{i=1}^{n} x_{i}}{n}=\frac{2}{\alpha \beta} \Gamma\left(\frac{1}{\alpha}\right) \sum_{j=0}^{\infty} \frac{(-1)^{j}}{(j+1)^{1 / \alpha}}, \\
& \frac{\sum_{i=1}^{n} x_{i}^{2}}{n}=\frac{4}{\alpha \beta^{2}} \Gamma\left(\frac{2}{\alpha}\right) \sum_{j=0}^{\infty} \frac{(-1)^{j}}{(j+1)^{2 / \alpha}} .
\end{aligned}
$$

4.3. Least Square Method. Least square estimation method involves the least squares regression to estimate the two parameters based on the linearized PHLD distribution function. For details of this procedure see Krishnaiah [10]. The basis of this method is the transformation of PHLD survival function

$$
\bar{F}(x)=\frac{2}{1+e^{\beta x^{\alpha}}},
$$

in the form

$$
\log x=\frac{-\log \beta}{\alpha}+\frac{1}{\alpha} \log \left[\log \left(\frac{2}{1-F(x)}-1\right)\right] .
$$

On putting $Y=\log x$ and $X=\log [\log ((2 /(1-F(x)))-1)]$, it becomes a linear function of $X$ and $Y$ in the form

$$
Y=\frac{1}{\alpha}(-\log \beta)+\frac{1}{\alpha} X .
$$

Note that $1 / \alpha$ is the slope of this equation and $(1 / \alpha) \cdot(-\log \beta)$ is the intercept.

Let $x_{1}<x_{2}<\cdots<x_{n}$ be the times of failure arranged in ascending order and $n$ is the sample size. Then $F\left(x_{i}\right)$ is estimated as in Zaka and Akhter [11], using Bernards' median rank method given by

$$
\widehat{F}\left(x_{i}\right)=\frac{i-0.3}{n+0.4} .
$$

\begin{tabular}{|c|c|c|c|c|c|}
\hline $\begin{array}{l}\text { Sample } \\
\text { size }\end{array}$ & $\begin{array}{c}\text { Parameters } \\
\alpha, \beta\end{array}$ & Method & $\begin{array}{c}\text { Estimates } \\
\widehat{\alpha}, \widehat{\beta}\end{array}$ & $\begin{array}{c}\text { K-S } \\
\text { distance }\end{array}$ & $p$ value \\
\hline \multirow[t]{2}{*}{$n=100$} & \multirow[t]{2}{*}{$0.5,0.8$} & MLE & $\begin{array}{l}0.5052 \\
0.8049\end{array}$ & 0.03 & 1.0 \\
\hline & & LSE & $\begin{array}{l}0.4973 \\
0.8069\end{array}$ & 0.04 & 1.0 \\
\hline \multirow{2}{*}{$n=50$} & \multirow{2}{*}{$0.5,0.8$} & MLE & $\begin{array}{l}0.5140 \\
0.8039\end{array}$ & 0.06 & 1.0 \\
\hline & & LSE & $\begin{array}{c}0.5008 \\
0.8122\end{array}$ & 0.06 & 1.0 \\
\hline \multirow{2}{*}{$n=20$} & \multirow{2}{*}{$0.5,0.8$} & MLE & $\begin{array}{l}0.5441 \\
0.7908\end{array}$ & 0.15 & 0.9831 \\
\hline & & LSE & $\begin{array}{l}0.5107 \\
0.8200\end{array}$ & 0.15 & 0.9831 \\
\hline \multirow[t]{2}{*}{$n=100$} & \multirow[t]{2}{*}{$1.2,1.0$} & MLE & $\begin{array}{l}1.1948, \\
1.0082\end{array}$ & 0.03 & 1.0 \\
\hline & & LSE & $\begin{array}{l}1.2218 \\
1.0012\end{array}$ & 0.03 & 1.0 \\
\hline \multirow[t]{2}{*}{$n=50$} & \multirow[t]{2}{*}{$1.2,1.0$} & MLE & $\begin{array}{l}1.2363 \\
0.9993\end{array}$ & 0.04 & 1.0 \\
\hline & & LSE & $\begin{array}{l}1.1968 \\
1.0147\end{array}$ & 0.04 & 1.0 \\
\hline \multirow{2}{*}{$n=20$} & \multirow[t]{2}{*}{$1.2,1.0$} & MLE & $\begin{array}{l}1.2968 \\
1.0093\end{array}$ & 0.1 & 1.0 \\
\hline & & LSE & $\begin{array}{l}1.2270 \\
1.0323\end{array}$ & 0.1 & 1.0 \\
\hline
\end{tabular}

Now the least square estimates of $\alpha$ and $\beta$ are

$$
\begin{aligned}
& \widehat{\alpha}=\frac{n \sum X_{i}^{2}-\left(\sum X_{i}\right)^{2}}{n \sum\left(X_{i} Y_{i}\right)-\sum X_{i} \sum Y_{i}}, \\
& \widehat{\beta}=e^{(-1 / n)\left(\widehat{\alpha} \cdot \sum Y_{i}-\sum X_{i}\right)},
\end{aligned}
$$

where $X_{i}$ and $Y_{i}$ are the values corresponding to the ordered failure times $x_{i}$.
TABle 1: Parameter estimates.

4.4. Numerical Examples. Samples of sizes 100, 50, and 20 are generated from PHLD for different values of parameters. The standard method of generation in R-programming is used for the generation of samples. We repeat this process 1000 times and compute simulated average, standard errors, confidence intervals, and coverage probabilities in each case. Comparison of the maximum likelihood estimation (MLE) and least square estimation (LSE) methods mentioned above is done. The computations are performed using R-programme and results are shown in Table 1. Kolmogorov-Smirnov (K-S) statistic and corresponding $p$ values are used for comparing the estimation methods. The $95 \%$ confidence intervals for the parameters using maximum likelihood estimates are also constructed in Table 2. Value of the K-S statistic is the same for both methods in most of the cases. Also $p$ value is the same in both cases. As there is no clear supremacy of a method over the other, we suggest MLE method since it is more prevalent and the estimates have better appealing properties. The distribution functions are considered for the generated sequence for given parameter values and also using estimated parameters and those functions are plotted in Figure 5. Similarly histograms and superimposed density curves for estimated values of the parameters are shown in Figure 6. From this we conclude that the above two methods of estimation are in agreement. Coverage probabilities for the parameters for different sample sizes are given in Table 3 and it is clear that they are higher in the case of LSE method. 
TABLE 2: Confidence interval for the parameters.

\begin{tabular}{|c|c|c|c|c|}
\hline Sample size & $\begin{array}{c}\text { Parameters } \\
\quad \alpha, \beta\end{array}$ & $\begin{array}{c}\text { Estimates } \\
\widehat{\alpha}, \widehat{\beta}\end{array}$ & $\begin{array}{c}\text { Confidence } \\
\text { intervals }\end{array}$ & MSE \\
\hline \multirow[t]{2}{*}{$n=100$} & 0.5 & 0.5052 & $\begin{array}{l}(0.4219 \\
0.5885)\end{array}$ & 0.0018 \\
\hline & 0.8 & 0.8049 & $\begin{array}{c}(0.60095 \\
1.0089)\end{array}$ & 0.0108 \\
\hline \multirow[t]{2}{*}{$n=50$} & 0.5 & 0.5140 & $\begin{array}{l}(0.3879 \\
0.6400)\end{array}$ & 0.0041 \\
\hline & 0.8 & 0.8039 & $\begin{array}{l}(0.5302 \\
1.0776)\end{array}$ & 0.0194 \\
\hline \multirow{2}{*}{$n=20$} & 0.5 & 0.5441 & $\begin{array}{l}(0.3176 \\
0.7707)\end{array}$ & 0.0133 \\
\hline & 0.8 & 0.7908 & $\begin{array}{c}(0.3631 \\
1.2186)\end{array}$ & 0.0476 \\
\hline \multirow[t]{2}{*}{$n=100$} & 1.2 & 1.1948 & $\begin{array}{l}(1.0142, \\
1.4293)\end{array}$ & 0.0112 \\
\hline & 1.0 & 1.0082 & $\begin{array}{l}(0.7976 \\
1.2049)\end{array}$ & 0.0107 \\
\hline \multirow[t]{2}{*}{$n=50$} & 1.2 & 1.2363 & $\begin{array}{l}(0.9437 \\
1.5288)\end{array}$ & 0.0228 \\
\hline & 1.0 & 0.9993 & $\begin{array}{l}(0.7065 \\
1.2920)\end{array}$ & 0.0223 \\
\hline \multirow[t]{2}{*}{$n=20$} & 1.2 & 1.2968 & $\begin{array}{l}(0.7858 \\
1.8088)\end{array}$ & 0.0679 \\
\hline & 1.0 & 1.0093 & $\begin{array}{c}(0.5248 \\
1.4938)\end{array}$ & 0.0611 \\
\hline
\end{tabular}

TABLE 3: Coverage probabilities for the parameters based on estimation methods.

\begin{tabular}{lccc}
\hline Method & Sample size & $\begin{array}{c}\text { Parameters } \\
\alpha=0.5, \beta=0.8\end{array}$ & $\begin{array}{c}\text { Parameters } \\
\alpha=1.2, \beta=1\end{array}$ \\
\hline MLE & \multirow{2}{*}{$n=100$} & $0.875,0.864$ & $0.834,0.832$ \\
LSE & & $0.942,0.955$ & $0.944,0.963$ \\
\hline MLE & \multirow{2}{*}{$n=50$} & $0.844,0.812$ & $0.734,0.724$ \\
LSE & & $0.946,0.95$ & $0.939,0.951$ \\
\hline MLE & \multirow{2}{*}{$n=20$} & $0.836,0.529$ & $0.575,0.593$ \\
LSE & & $0.94,0.956$ & $0.942,0.955$ \\
\hline
\end{tabular}

\section{Extensions of PHLD}

Several types of extensions are possible using this distribution. Some of them are very much related with already existing distributions in the literature. These are discussed in this section.

5.1. Log Power Half-Logistic Distributions. Consider the distribution of some log transformations and we call the distributions obtained as log Power Half-Logistic Distributions. When $Y=e^{X}$ and $X$ has PHLD, then

$$
f(y)=\frac{2 \alpha \beta e^{\beta(\log y)^{\alpha}}(\log y)^{\alpha-1}}{y\left(1+e^{\beta(\log y)^{\alpha}}\right)^{2}}, \quad y>1 .
$$

This distribution is called as log positive Power Half-Logistic Distribution.

In a similar way we define the distribution of $Y=e^{-x}$ as $\log$ negative Power Half-Logistic Distribution with support $0<y<1$.

$$
\begin{aligned}
& \text { If } Y=e^{x^{\alpha}} \text {, where } X \sim \operatorname{PHLD} \text {, the pdf of } Y \text { is given by } \\
& \qquad g(y)=\frac{2}{(1+y)^{2}}, \quad y>1 .
\end{aligned}
$$

Similarly the pdf of $Z=e^{-x^{\alpha}}$ is

$$
g(z)=\frac{2}{(1+z)^{2}}, \quad 0<z<1 .
$$

So we get two distributions with the same structure but defined at two disjoint intervals $[1, \infty)$ and $[0,1]$ which is the characteristic of a nonnegative random variable with respect to log transformations. These are having the same form as we transform half-logistic distribution by the transformations $Y=e^{x}$ and $Z=e^{-x}$.

Immediately, we have the following result, which may be exploited for generating random variables from PHLD.

Result 4. If $X \sim U(0,1)$, then the random variable $Z=$ $[(1 / \beta) \log (a-X) / X]^{1 / \alpha}$ has truncated PHLD (TPHLD).

Proof. If $X \sim U(0,1)$, then

$$
\begin{aligned}
P(Z \geq z) & =P\left(\left[\left(\frac{1}{\beta}\right) \log \left(\frac{a-X}{X}\right)\right]^{1 / \alpha} \geq z\right) \\
& =\frac{a}{1+e^{\beta z^{\alpha}}},
\end{aligned}
$$

which is a new distribution with density function

$$
\begin{aligned}
f(x)= & \frac{\alpha a \beta e^{\beta x^{\alpha}} x^{\alpha-1}}{\left(1+e^{\beta x^{\alpha}}\right)^{2}} \\
& x>\left[\frac{1}{\beta} \log (a-1)\right]^{1 / \alpha}, \alpha>0, \beta>0, a \geq 2 .
\end{aligned}
$$

We call this as Truncated Power Half-Logistic Distribution (TPHLD). Note that when $\alpha=1$, we have the Truncated Half-Logistic Distribution; when $a=2, \alpha=1$, it gives HLD; and when $a=2, \alpha>0$, it gives PHLD.

5.2. Families of Distributions Generated from PHLD. We have, in the literature, quite a few families of distributions generated from Beta and Gamma distributions (see $[7,12,13])$. This type of distributions is generalizations of many existing families. Here we generate families of distributions from the PHLD. A detailed study of this type of distributions, its properties, applications, and so forth is not attempted in this paper for brevity, but would be carried out in future. 


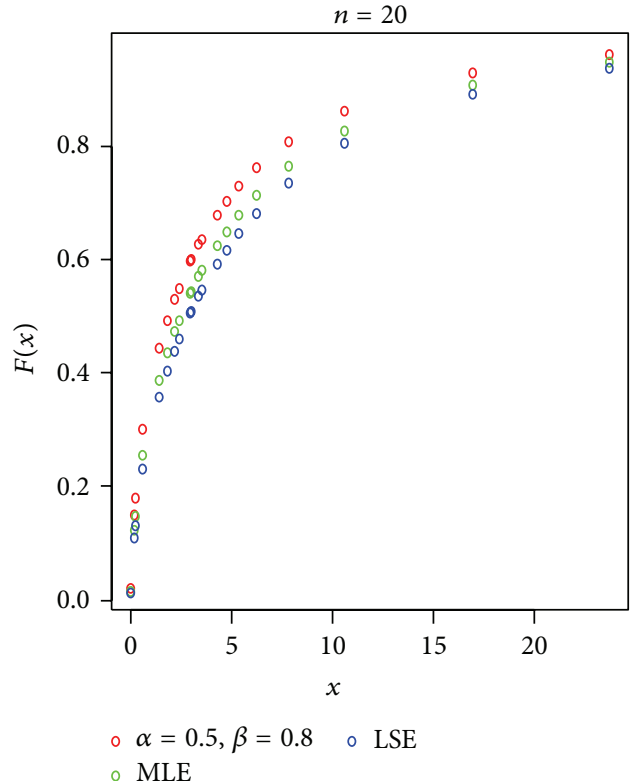

(a)

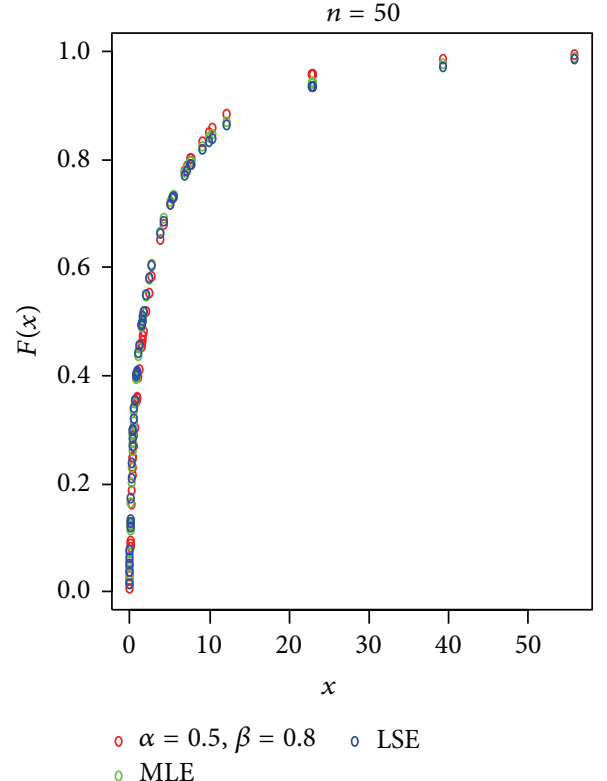

(b)

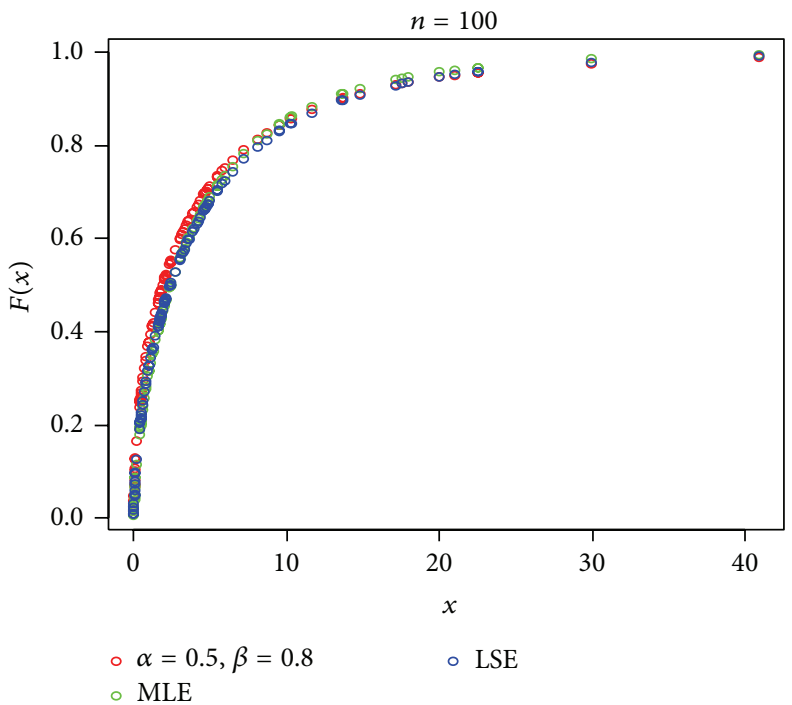

(c)

FIgURE 5: Distribution functions when $n=20,50,100$.

Define a new transformation

$$
G_{1}(x)=\int_{0}^{\phi(x)} d(H(x)),
$$

where $H(x)$ is the distribution function of PHLD or its generalizations and $\phi(x)$ takes different forms of $\bar{G}(x)$, the survival function of a random variable. This transformation gives us very interesting results as summarized in Table 4. Note that when $\beta=1, \bar{G}(x)$ in Result number 1 in Table 4 has Marshall and Olkin [14] form with parameter 2. So a general structure is needed for constructing Marshall-Olkin form with parameter $\alpha$. This is explained in the following remark.
Remark 1. Consider a new distribution, called General Power Half-Logistic Distribution (GPHLD), by adding a skewness parameter $\gamma$,

$$
h(x)=(1+\gamma) \frac{\gamma \beta \alpha e^{\beta x^{\alpha}} x^{\alpha-1}}{\left(1+\gamma e^{\beta x^{\alpha}}\right)^{2}} ; \quad x>0, \alpha>0 .
$$

The survival function is

$$
\bar{H}(x)=\frac{1+\gamma}{1+\gamma e^{\beta x^{\alpha}}} .
$$



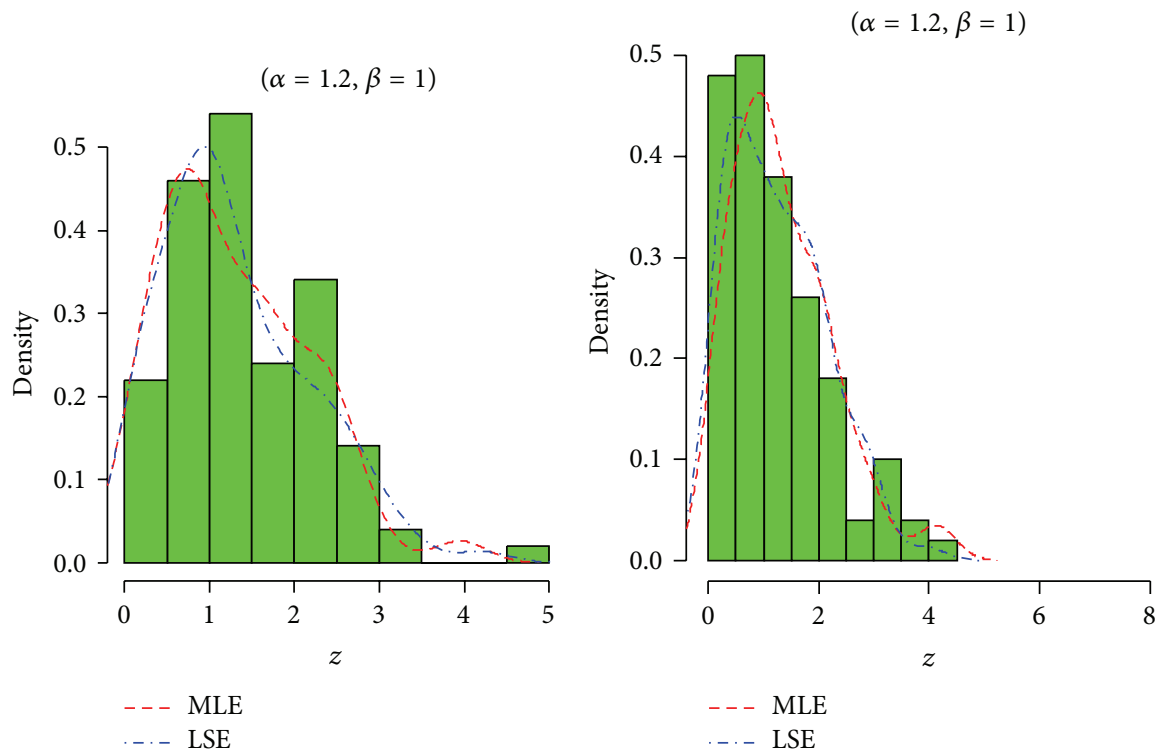

(a)
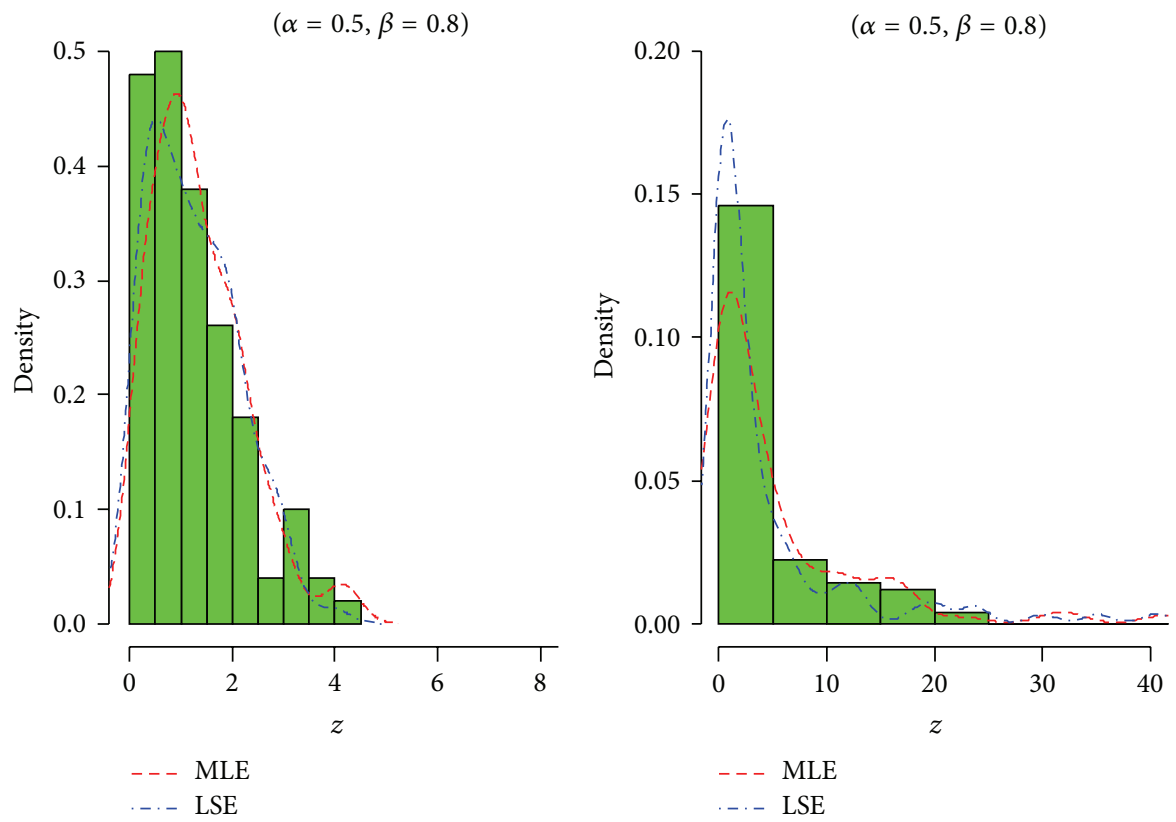

(c)

(d)

FIGURE 6: Fitted density curves based on estimation.

Using this in (25) we get a new family of life distributions, with survival function

$$
\bar{H}(x)=\frac{\bar{G}(x)^{\beta}(1+\gamma)}{\gamma+\bar{G}(x)^{\beta}}
$$

which can be considered as a generalization of Marshall and Olkin [14] (M-O) form with survival function $\alpha \bar{F}(x) /(1-$ $\bar{\alpha} \bar{F}(x)), \alpha>0$. Let $\beta=1$ in (28); then corresponding density function is given by $h(x)=(1+\gamma) \gamma g(x) /(\gamma+\bar{G}(x))^{2}$ and hazard rate is $(\gamma /(\gamma+\bar{G}(x))) r(x)$ where $r(x)$ is the hazard rate function of $g(x)$. We can see that the parameters $\gamma$ in (28) and $\alpha$ in M-O are related as $\gamma=1 /(\alpha-1)$.

Interestingly we have noted that (27) is the Weibullgeometric distribution introduced by Barreto-Souza et al. [15] with parameter $\theta$ in $[-1, \infty)$.

Remark 2. Result number 3 of Table 4 is obtained by taking the $\gamma$ th power of distribution function of PHLD (called as Type I PHLD) which is the same as in Cordeiro et al. [7]. 
TABLE 4: Families of distributions generated.

\begin{tabular}{lccc}
\hline $\begin{array}{l}\text { Result } \\
\text { number }\end{array}$ & $\begin{array}{c}H(x), x>0, \\
\alpha>0, \beta>0\end{array}$ & $\phi(x)$ & $\overline{G_{1}}(x)$ \\
\hline 1 & $1-\frac{2}{1+e^{\beta x^{\alpha}}}$ & $(-\log \bar{G}(x))^{1 / \alpha}$ & $\frac{2 \bar{G}^{\beta}}{1+\bar{G}^{\beta}}$ \\
\hline 2 & $1-\frac{2}{1+e^{\beta x^{\alpha}}}$ & $\bar{G}$ & $\frac{2}{1+e^{\beta(G / \bar{G})^{\alpha}}}$ \\
\hline 3 & {$\left[1-\frac{2}{1+e^{\beta x^{\alpha}}}\right]^{\gamma}$} & $(-\log \bar{G}(x))^{1 / \alpha}$ & $1-\left[\frac{1-\bar{G}(x)^{\beta}}{1+\bar{G}(x)^{\beta}}\right]^{\gamma}$ \\
\hline 4 & $1-\left(\frac{2}{1+e^{\beta x^{\alpha}}}\right)^{\gamma}$ & $(-\log \bar{G}(x))^{1 / \alpha}$ & $\left.\frac{2 \bar{G}(x)^{\beta}}{1+\bar{G}(x)^{\beta}}\right]^{\gamma}$ \\
\hline
\end{tabular}

TABLE 5: Fitting based on data set 1 .

\begin{tabular}{lcccr}
\hline Distribution & Parameter estimates & Log likelihood & K-S distance & $p$ value \\
\hline Weibull & $\widehat{\alpha}=2.08, \widehat{\beta}=0.0001$ & -113.6902 & 0.1304 & 0.9897 \\
PHLD & $\widehat{\alpha}=1.77, \widehat{\beta}=0.0006$ & -114.0720 & 0.2609 & 0.4143 \\
Type I HLD & $\widehat{\alpha}=3.41, \widehat{\beta}=0.0350$ & -113.0407 & 0.2174 & 0.6487 \\
GPHLD & $\widehat{\alpha}=2.01, \widehat{\beta}=0.0002, \widehat{\gamma}=4.96$ & -113.7000 & 0.1304 & 0.9897 \\
\hline
\end{tabular}

TABLE 6: Fitting based on data set 2.

\begin{tabular}{lcccc}
\hline Distribution & Parameter estimates & Log likelihood & K-S distance & $p$ value \\
\hline Weibull & $\widehat{\alpha}=5.78, \widehat{\beta}=0.06$ & -15.2070 & 0.2063 & 0.1367 \\
PHLD & $\widehat{\alpha}=5.05, \widehat{\beta}=0.13$ & -13.8273 & 0.1587 & 0.4055 \\
Type I HLD & $\widehat{\alpha}=5.04, \widehat{\beta}=0.13$ & -13.8300 & 0.1587 & 0.4055 \\
GPHLD & $\widehat{\alpha}=3.202, \widehat{\beta}=0.695, \widehat{\gamma}=0.064$ & -12.0336 & 0.0952 & 0.9375 \\
\hline
\end{tabular}

Remark 3. $\overline{G_{1}}(x)$ in Result number 4 of Table 4 is a member of the Lehmann family of distributions and this is Type II PHLD.

\section{Applications}

In this section we use three sets of real-life data to fit the distributions. The analysis is done using R-programming software. The first set is discussed by Gupta and Kundu [16] in the fitting of exponentiated exponential distribution.

Data Set 1. The first data set is taken from Lawless [17, page 98]. The data are the number of million revolutions before failure for each of the 23 ball bearings in the life test and they are $17.88,28.92,33.00,41.52,42.12,45.60,48.80,51.84,51.96$, $54.12,55.56,67.80,68.64,68.64,68.88,84.12,93.12,98.64$, $105.12,105.84,127.92,128.04$, and 173.40. We consider the Weibull, Type I HLD (Kantam et al. [8]), PHLD, and GPHLD for this particular data set.

The likelihood value as noted in Table 5 is greatest for Type 1 HLD and also based on $p$ values of K-S statistic, we conclude that Type 1 HLD is a good fit for the data.

Data Set 2. This data set is from Smith and Naylor [18] representing strengths of $1.5 \mathrm{~cm}$ glass fibres. The data set is $0.55,0.93,1.25,1.36,1.49,1.52,1.58,1.61,1.64,1.68,1.73,1.81,2$,
$0.74,1.04,1.27,1.39,1.49,1.53,1.59,1.61,1.66,1.68,1.76,1.82$, $2.01,0.77,1.11,1.28,1.42,1.5,1.54,1.6,1.62,1.66,1.69,1.76$, $1.84,2.24,0.81,1.13,1.29,1.48,1.5,1.55,1.61,1.62,1.66,1.7,1.77$, $1.84,0.84,1.24,1.3,1.48,1.51,1.55,1.61,1.63,1.67,1.7,1.78$, and 1.89 (see [19]). They have fitted different distributions to this data set. When we use this data set for the four distributions, Weibull, Type I HLD, PHLD, and GPHLD, the results are as follows.

Morais and Barreto-Souza [19] have shown that the Weibull-geometric distribution is better fit to this data set. The log-likelihood values, K-S distance, and $p$ values in Table 6 reveal that GPHLD is better than the other three models (see Remark 1 in this context).

Data Set 3. This data set is of camber of 497 lead wires taken from Leone et al. [20]. Cooray et al. [21] considered this data and fitted folded logistic distribution. They got log-likelihood $=-1698.24$, K-S distance $=0.06$, and $p$ value $=0.32$. Results are illustrated in Table 7 and it is clear that PHLD is the most suitable for this data set.

\section{Conclusions}

A new distribution on the positive real line is constructed using power transformation on half-logistic distribution. 
TABLE 7: Fitting based on data set 3.

\begin{tabular}{|c|c|c|c|c|}
\hline Distribution & Parameter estimates & Log likelihood & K-S distance & $p$ value \\
\hline Weibull & $\widehat{\alpha}=1.860, \widehat{\beta}=0.0059$ & -1692.670 & 0.0795 & 0.10 \\
\hline PHLD & $\widehat{\alpha}=1.595, \widehat{\beta}=0.0180$ & -1692.180 & 0.0785 & 0.10 \\
\hline Type I HLD & $\widehat{\alpha}=2.095, \widehat{\beta}=0.1450$ & -1698.953 & 0.0845 & 0.06 \\
\hline GPHLD & $\widehat{\alpha}=1.824, \widehat{\beta}=0.0069, \widehat{\gamma}=9.934$ & -1692.484 & 0.0986 & 0.02 \\
\hline
\end{tabular}

Analytical properties, some characterizations, and estimation of the parameters are done. New families of distributions are generated from this new distribution which generalizes many existing families of distributions. Applications are discussed with the help of three data sets. The properties, characteristics, and applications of the newly generated families of distributions are further topics for future research work. Some of these families are generated using the odds function.

\section{Conflict of Interests}

The author declares that there is no conflict of interests regarding the publication of this paper.

\section{Acknowledgment}

The author is highly grateful to referees for their valuable comments and suggestions for improving the paper.

\section{References}

[1] N. Balakrishnan, "Order statistics from the half logistic distribution," Journal of Statistical Computation and Simulation, vol. 20, no. 4, pp. 287-309, 1985.

[2] N. Balakrishnan, Handbook of the Logistic Distribution, vol. 123 of Statistics: A Series of Textbooks and Monographs, Marcel Dekker, New York, NY, USA, 1992.

[3] B. Srinivasa Rao, S. Nagendram, and K. Rosaiah, "Exponential Halflogistic additive failure rate model," International Journal of Scientific and Research Publications, vol. 3, no. 5, pp. 1-10, 2013.

[4] A. K. Olapade, "On Characterizations of the Half Logistic Distribution,” Interstat, February Issue, Number 2, 2003, http://interstat.statjournals.net/.

[5] A. K. Olapade, "On type III generalized half logistic distribution," Journal of the Iranian Statistical Society, vol. 13, no. 1, 2008.

[6] A. K. Olapade, "The type I generalized half logistic distribution," Journal of Iranian Statistical Society, vol. 13, no. 1, pp. 69-88, 2014.

[7] G. M. Cordeiro, M. Alizadeh, and E. M. M. Ortega, "The exponentiated half-logistic family of distributions: properties and applications," Journal of Probability and Statistics, vol. 2014, Article ID 864396, 21 pages, 2014.

[8] R. R. L. Kantam, V. Ramakrishna, and M. S. Ravikumar, "Estimation and testing in Type I generalized half logistic distribution," Journal of Modern Applied Statistical Methods, vol. 12, no. 1, pp. 198-206, 2013.

[9] A. W. Marshall and I. Olkin, Life Distributions: Structure of Nonparametric, Semiparametric, and Parametric Families, Springer Series in Statistics, Springer, New York, NY, USA, 2007.
[10] K. Krishnaiah, Applied Statistical Quality Control and Improvement, Eastern Economy Edition, PHL Learning Private Limited, 2014.

[11] A. Zaka and A. S. Akhter, "Methods for estimating the parameters of the power function distribution," Pakistan Journal of Statistics and Operation Research, vol. 9, no. 2, pp. 213-224, 2013.

[12] N. Eugene, C. Lee, and F. Famoye, "Beta-normal distribution and its applications," Communications in Statistics-Theory and Methods, vol. 31, no. 4, pp. 497-512, 2002.

[13] K. Zografos and N. Balakrishnan, "On families of betaand generalized gamma-generated distributions and associated inference," Statistical Methodology, vol. 6, no. 4, pp. 344-362, 2009.

[14] A. W. Marshall and I. Olkin, "A new method for adding a parameter to a family of distributions with application to the exponential and Weibull families," Biometrika, vol. 84, no. 3, pp. 641-652, 1997.

[15] W. Barreto-Souza, A. L. De Morais, and G. M. Cordeiro, "The Weibull-geometric distribution," Journal of Statistical Computation and Simulation, vol. 81, no. 5, pp. 645-657, 2011.

[16] R. D. Gupta and D. Kundu, "Exponentiated exponential family: an alternative to gamma and Weibull distributions," Biometrical Journal, vol. 43, no. 1, pp. 117-130, 2001.

[17] J. F. Lawless, Statistical Models and Methods for Lifetime Data, John Wiley \& Sons, New York, NY, USA, 2nd edition, 2003.

[18] R. L. Smith and J. C. Naylor, "A comparison of maximum likelihood and Bayesian estimators for the three-parameter Weibull distribution," Journal of the Royal Statistical Society. Series C. Applied Statistics, vol. 36, no. 3, pp. 358-369, 1987.

[19] A. L. Morais and W. Barreto-Souza, "A compound class of Weibull and power series distributions," Computational Statistics and Data Analysis, vol. 55, no. 3, pp. 1410-1425, 2011.

[20] F. C. Leone, L. S. Nelson, and R. B. Nottingham, "The folded normal distribution," Technometrics, vol. 3, no. 4, pp. 543-550, 1961.

[21] K. Cooray, S. Gunasekera, and M. M. A. Ananda, “The folded logistic distribution," Communications in Statistics-Theory and Methods, vol. 35, no. 1-3, pp. 385-393, 2006. 


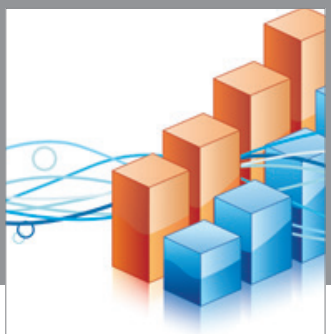

Advances in

Operations Research

vatem alat4

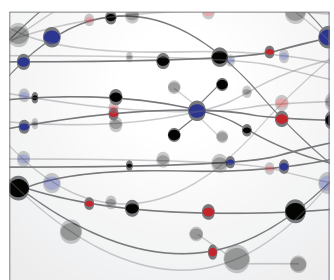

\section{The Scientific} World Journal
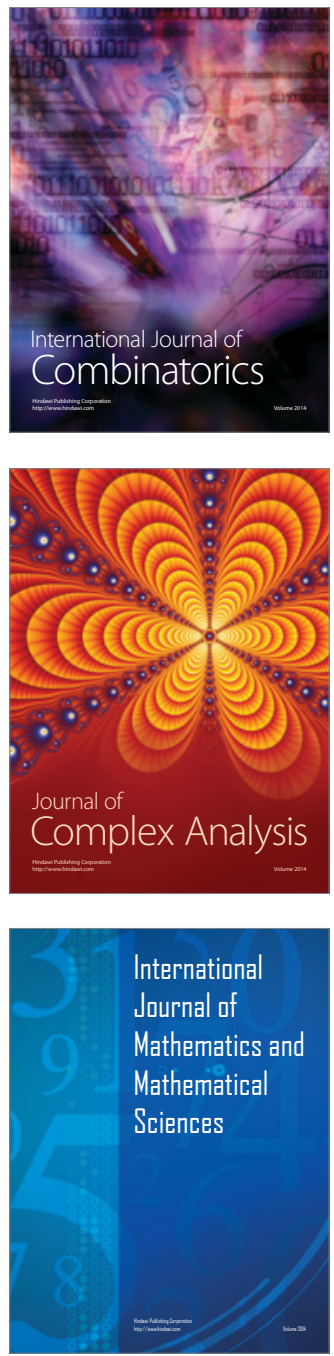
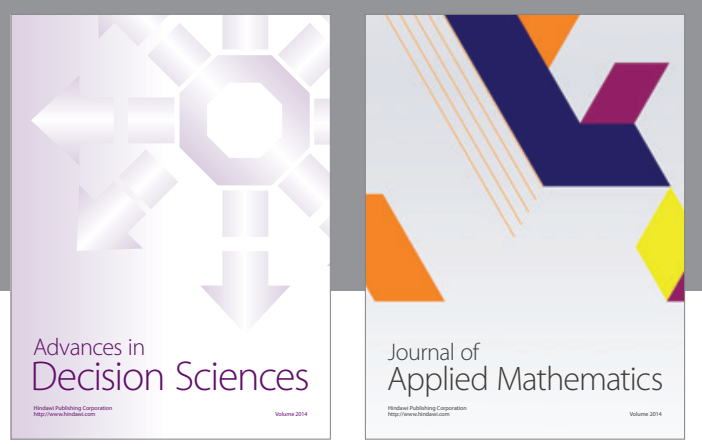

Algebra

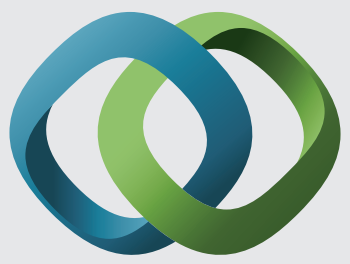

\section{Hindawi}

Submit your manuscripts at

http://www.hindawi.com
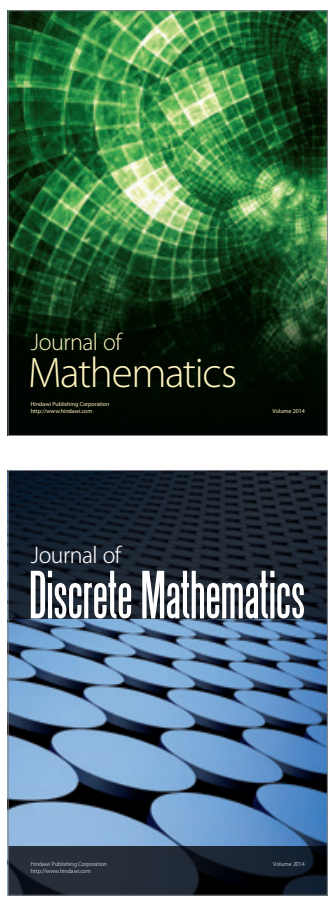

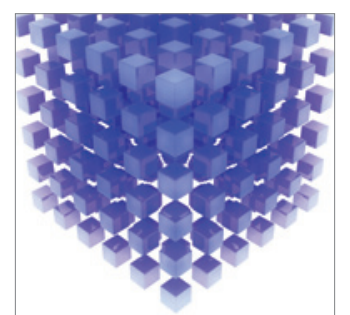

Mathematical Problems in Engineering
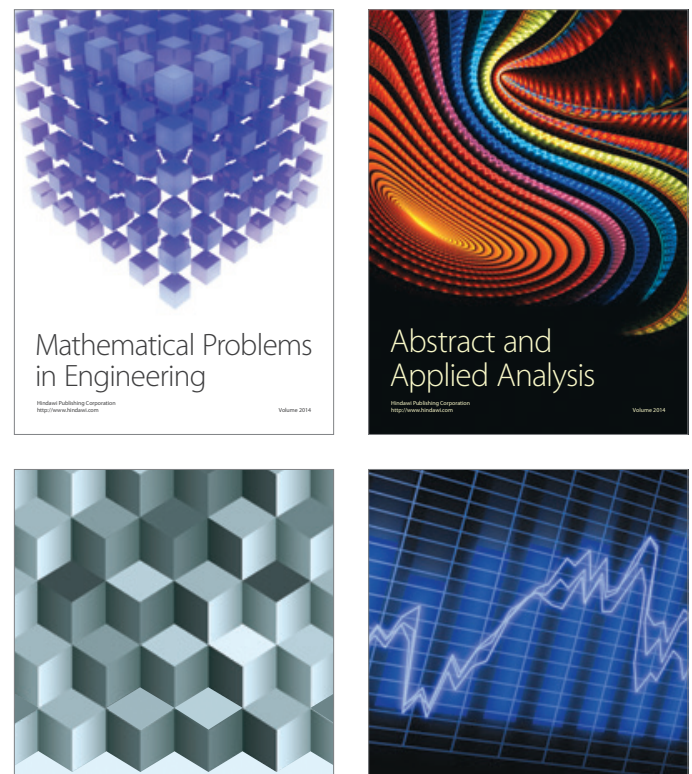

Journal of

Function Spaces

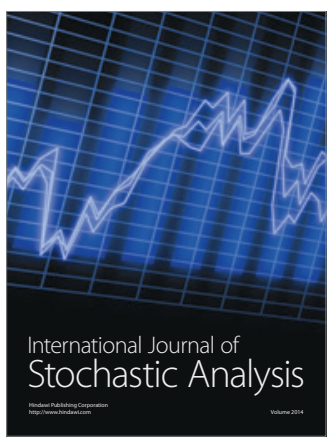

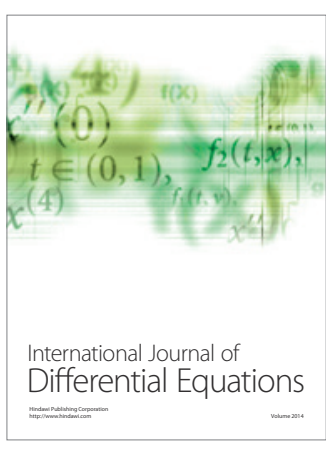
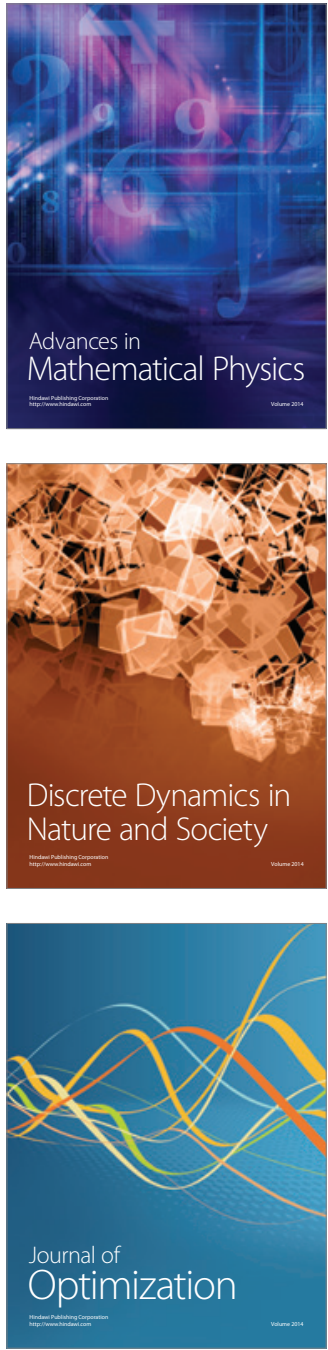